\title{
The role of robotic partial cystectomy in a patient with metastatic primary adenocarcinoma of the bladder
}

\author{
Katherine James, Nikhil Vasdev, James M Adshead \\ Department of Urology, Hertfordshire and South Bedfordshire Robotic Urological Cancer Centre, Lister Hospital, Stevenage, UK \\ Received January 04, 2015; Revised January 17, 2015; Accepted January 19, 2015; Published Online February 06, 2015
}

\section{Case Report}

\begin{abstract}
Treatment of urachal adenocarcinoma (UA) of the urinary bladder has typically been with radical cystectomy (RC) but more conservative approaches are gaining popularity. Here we present the case of a female patient with metastatic primary bladder UA who was treated with robotic partial cystectomy (RPC) and adjuvant chemotherapy; she is alive with no evidence of disease recurrence or metastatic disease at 5 years. This case provides some of the longest follow-up after RPC to date thereby demonstrating that RPC is a safe and oncologically viable treatment for selected patients even several years after definitive treatment. Patients undergoing RPC benefit from the reduced morbidity associated with this less radical treatment whilst enjoying similarly successful oncological outcomes to RC.
\end{abstract}

Keywords: Robotic Partial Cystectomy; Adenocarcinoma Bladder; Metastasis; Outcome

\section{Introduction}

Urachal adenocarcinoma (UA) of the urinary bladder is a rare and aggressive tumour that occurs at the dome or anterior wall of the bladder. ${ }^{1}$ It is typically associated with an extremely poor prognosis with many patients found to have distant metastases at the time of diagnosis. ${ }^{2}$ The gold standard of treatment for muscle invasive bladder cancer is radical cystectomy ${ }^{3,4}$ but the alternative of partial cystectomy (PC) has gained popularity and has been shown to provide comparable 5-year survival rates to patients treated with radical cystectomy. ${ }^{1,5}$

In 2006 Milhoua's team published the successful outcome of a patient with primary malignant UA who underwent a laparoscopic extended PC and en bloc removal of the urachus and umbilicus; this patient remained disease-free at 18 months. ${ }^{5}$ In 2010 Allaparthi's team published the successful short-term outcome of a patient with UA who underwent robotic PC (RPC). ${ }^{6}$ Here we present the case of a patient with metastatic UA who underwent robotic PC who is alive with no evidence of disease recurrence or metastatic disease at 5 years.

\section{Case presentation}

A 49-year-old lady was diagnosed with a large urachal adenocarcinoma with metastatic nodal disease (T4bN3) in 2010, having been investigated by gynaecology for 'vaginal bleeding' for 6 months (Figures 1 and 2).

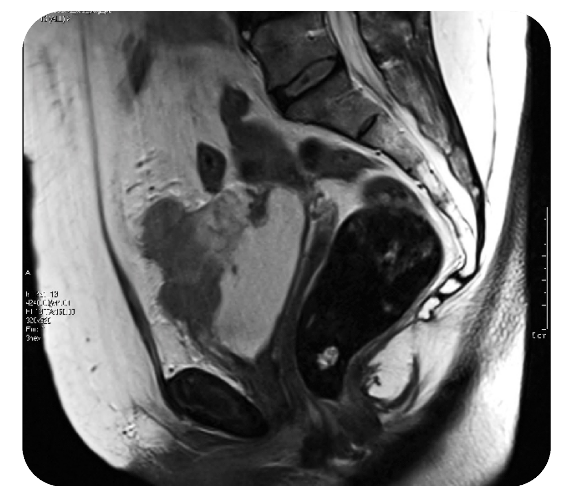

FIG. 1: Initial MRI scans showing this patient's large urachal adenocarcinoma.

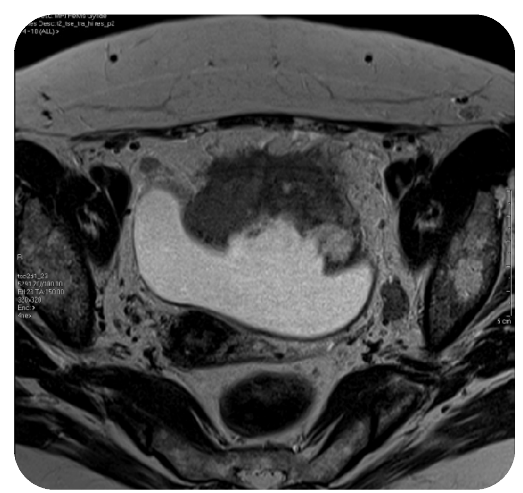

FIG. 2: Initial MRI scans showing this patient's large urachal adenocarcinoma. 
The tumour was invading the omentum and she underwent robotic PC, with en bloc removal of a patch of omentum in June 2008. 24 pelvic nodes were removed, with 3 nodes on the left found to be positive. A left superficial groin dissection revealed 1 positive node, which measured over $5 \mathrm{~cm}$. Estimated blood loss (EBL) was $150 \mathrm{ml}$, and she was discharged after three nights in hospital. She subsequently had four cycles of adjuvant oxaliplatin and capecitabine and she remains well with no recurrence or evidence of metastatic disease. Her pre- and post-treatment PET scans are shown below (Figures 3 and 4); these show the remarkable and complete response to treatment.

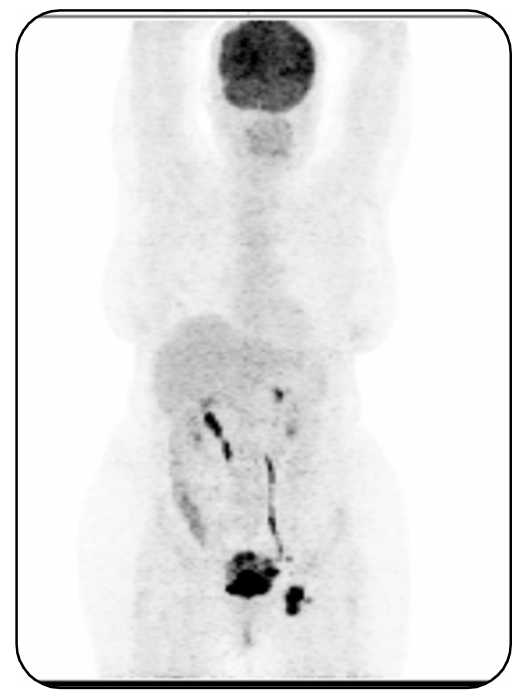

FIG. 3: Initial PET scan in March 2010 (pre-treatment).

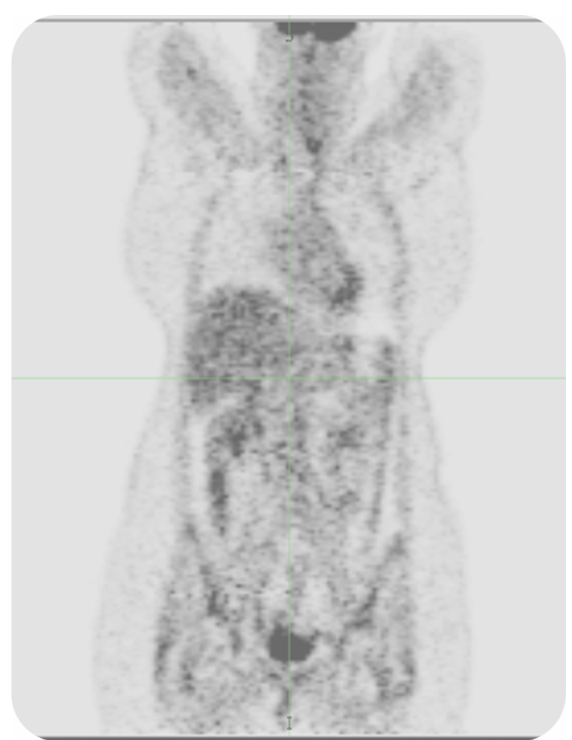

FIG. 4: PET scan 42 months post-surgery and chemotherapy showing complete response to treatment.

\section{Discussion}

This case shows that the more conservative treatment option of robotic partial cystectomy can provide successful onco- logical results even for patients with advanced metastatic disease. Our case demonstrates that these successful oncological results are also seen several years after treatment, with previously published cases only reporting data of follow-up of several months. That RPC can provide comparable oncological results to radical cystectomy is an important advance in urological, and robotic urological, practice for several reasons. First, by undergoing RPC patients avoid the long-term consequences of requiring a urinary diversion system, such as psychological and cosmetic issues, as well as the risk of hyperchloraemic metabolic acidosis. The early post-operative complications associated with $\mathrm{RC}$, such as dehydration, are also avoided; these have been quoted as occurring in up to $28 \%$ of patients undergoing RC. ${ }^{7}$ Second, RPC provides a curative treatment option for patients with too poor a functional status to undergo radical cystectomy. ${ }^{3}$ Third, as is the case for robotic surgery in general, robotic PC is associated with a faster postoperative recovery, less postoperative pain and improved post-operative cosmetic appearance. 8,9

This case also demonstrates a good response of UA to chemotherapy, contrary to what has been previously shown. ${ }^{1,10}$ Further research is needed to establish the role of adjuvant chemotherapy in treating UA.

One of the main concerns regarding the use of $\mathrm{PC}$ has been of recurrence within the bladder, distal ureter and the prostate in men, with two large studies recording local recurrence rates of up to $35 \% .{ }^{6}$ However, the largest, and more recent, study of UA to date reported more modest recurrence rates; $15 \%$ of patients with non-metastatic UA developed local recurrence, and this was usually after partial cystectomy. ${ }^{1}$ However, there was no difference in survival rates between patients who underwent partial cystectomy versus radical cystectomy.

UA is a rare pathology with the details of around only 400 cases previously published. Only a few of these patients have been treated with robotic partial cystectomy, and there is currently only short-term follow-up of these patients, albeit good responses to treatment in the short-term. Here we provide data to show that patients with aggressive UA are undergoing less invasive surgery and remaining disease-free without recurrence several years down the line.

\section{Conclusion}

There is an increasing body of evidence in the literature to suggest that robotic partial cystectomy is an oncologically safe and feasible curative treatment option for patients with urachal adenocarcinoma, and that its results are comparable with radical cystectomy. This case adds some further evidence to the literature but the success of RPC for this rare malignancy needs to be verified with further studies and more long-term follow-up. 


\section{Conflict of interest}

The authors declare that they have no conflicts of interest. The authors alone are responsible for the content and writing of the paper.

\section{References}

1. Ashley RA, Inman BA, Sebo TJ, et al. Urachal carcinoma: clinicopathologic features and long-term outcomes of an aggressive malignancy. Cancer 2006; 107:712-20.

2. Henly DR, Farrow GM, Zincke H. Urachal cancer: role of conservative surgery. Urology 1993; 42:635-9.

3. Knoedler JJ, Boorjian SA, Kim SP, et al. Does partial cystectomy compromise oncologic outcomes for patients with bladder cancer compared to radical cystectomy? A matched case-control analysis. $J$ Urol 2012; 188:1115-9.

4. Vasdev N, Lamb B, Lane T, et al. Robotic Cystectomy: Important considerations before commenc- ing the procedure independently. Int J Cancer Ther Oncol 2013; 1:01017.

5. Milhoua PM, Knoll A, Bleustein CB, Ghavamian R. Laparoscopic partial cystectomy for treatment of adenocarcinoma of the urachus. Urology 2006; 67:423.e15-423.e17.

6. Allaparthi S, Ramanathan R, Balaji KC. Robotic partial cystectomy for bladder cancer: a single-institutional pilot study. J Endourol 2010; 24:223-7.

7. Stein JP, Skinner DG. Radical cystectomy for invasive bladder cancer: long-term results of a standard procedure. World J Urol 2006; 24:296-304.

8. Spiess PE, Correa JJ. Robotic assisted laparoscopic partial cystectomy and urachal resection for urachal adenocarcinoma. Int Braz J Urol 2009; 35:609.

9. Chan KE, Vasdev N. Current status of robotic training in the UK - a trainees perspective. Int J Cancer Ther Oncol 2014; 2:02013.

10. Siefker-Radtke A. Urachal adenocarcinoma: a clinician's guide for treatment. Semin Oncol 2012; 39:619-24. 